\title{
O conceito de espécie no ensino de biologia: reflexões sobre seu estatuto ontológico com foco na biodiversidade
}

\author{
The concept of species on biology teaching: reflections about his ontological \\ status focusing on biodiversity
}

\author{
LUCIANO NEVES DA SILVA ${ }^{1}$ \\ FERNANDA APARECIDA MEGLHIORATTI ${ }^{2}$
}

\begin{abstract}
Resumo: Ao oferecer uma ontologia para descrever a diversidade e os processos que ocorrem na natureza, a importância da espécie é reforçada por biólogos e filósofos. As espécies são apresentadas como unidade da diversidade e de evolução. As espécies tradicionalmente foram imaginadas como classes, no entanto, recentemente foi proposto que as espécies devem ser consideradas entidades individuais, entendidas como totalidades concretas e coesas, delimitadas no espaço e no tempo. As discussões epistemológicas do conceito de espécie biológica são importantes também para o Ensino de Biologia. Os documentos curriculares para o Ensino Médio preconizam que o Ensino de Biologia deve proporcionar ao estudante a possibilidade de participar dos debates atuais que necessitam de conhecimentos biológicos. Assim, o professor ao abordar com seus alunos os conteúdos de classificação dos seres vivos, a sistemática e o conceito de espécie também precisa abordar a diversidade de seres vivos no planeta, conteúdos estes associados com temáticas evolucionistas e a abordagem da biodiversidade. O Conceito Biológico de Espécie que é amplamente aceito na Biologia Evolutiva atua como uma linha de condução para o entendimento do conhecimento das espécies e sua relação com a preservação da biodiversidade. Exposto brevemente a temática do trabalho, o presente texto buscou refletir o conceito de espécie, em especial, o estatuto ontológico do Conceito Biológico de Espécie, bem como sua onipresença nos livros didáticos do Ensino Médio, apresentando também os documentos nacionais de Educação e o modo como orientam o ensino a respeito de espécies.
\end{abstract}

Palavras-Chave: Conceito Biológico de Espécie. Ontologia das Espécies. Livro Didático. Epistemologia da Biologia.

\begin{abstract}
By offering an ontology to describe the diversity and processes that occur in nature, the importance of the species is reinforced by biologists and philosophers. Species are presented as a unit of diversity and evolution. Species have traditionally been thought of as classes, however, it has recently been proposed that species should be considered individual entities, understood as concrete and cohesive totalities, delimited in space and time. Epistemological discussions of the concept of biological species are also important for the teaching of biology. The curricular documents for High School recommend that the Teaching of Biology should provide the student with the possibility of participating in the current debates that require biological knowledge. Thus, the teacher when approaching with his students the content of classification of living beings, the systematics and the concept of species also needs to address the diversity of living beings on the planet, content associated with evolutionary themes and the approach to biodiversity. The Biological Concept of Species, which is widely accepted in Evolutionary Biology, acts as a guideline for understanding species knowledge and its relationship with the preservation of biodiversity. Briefly exposed the theme of the work, this text sought to reflect the concept of species, in
\end{abstract}

\footnotetext{
${ }^{1}$ Universidade Estadual do Oeste do Paraná. E-mail: lucnevesmn@gmail.com

${ }^{2}$ Universidade Estadual do Oeste do Paraná. E-mail: Fernanda.meglhioratti@gmail.com
} 
particular, the ontological status of the Biological Concept of Species, as well as its omnipresence in high school textbooks, also presenting the national documents of Education and the way how they guide teaching about species.

Keywords: Biological Concept of Species; Species Ontology; Textbook; Epistemology of Biology.

\section{Introdução}

Os biólogos e filósofos acreditam que o conceito de espécie é importante, pois oferece uma ontologia para descrever a diversidade presente na natureza e para processos postulados para operar na natureza, por exemplo, a especiação, a competição, entre tantos outros (CRACRAFT, 1987). As espécies também são descritas como a unidade da diversidade (CLARIDGE; DAWAH, 1997) e como a principal unidade da evolução (RIDLEY, 2006; MAYR, 2000a).

O conceito de espécie possui importância significativa em diversas disciplinas. $\mathrm{Na}$ sistemática e taxonomia, de modo especial, os biólogos se debruçam sobre exemplares de espécimes, valendo-se de muitos recursos, que vão desde simples caracteres morfológicos até complexas análises moleculares e genéticas que podem promover a melhor descrição e maior parcimônia para a definição de uma espécie na natureza.

O conceito de espécie é importante tanto para a produção do conhecimento científico em laboratórios e centros de pesquisas de ciências quanto para o aprendizado de conteúdos da disciplina de Biologia. O conceito de espécie está presente nas orientações curriculares de documentos oficiais de Educação para o Ensino Médio (BRASIL, 20o6a), sendo também apresentado nos livros didáticos do Ensino Médio utilizados nas escolas brasileiras (SILVA, 2018). Nos livros didáticos, os conhecimentos acadêmicos são adaptados para o conhecimento escolar, de modo que possam ser compreendidos e apreendidos pelos estudantes, esta adaptação corresponde ao processo de transposição didática, apresentada em Chevallard (1982).

A ampla discussão acerca do conceito de espécie no ambiente acadêmico traduz a relevância da temática, suscitando novos debates a cada dia, promovendo sob a ótica biológica, epistemológica e filosófica a reflexão de temas, tais como: a história e desenvolvimento do conceito de espécie (ZACHOS, 2016; WILKINS, 2010); a mais abrangente definição de espécies proposta por um conceito (DE QUEIROZ, 2005a); o problema da espécie ${ }^{3}$ (RICHARDS, 2010; MAYR, 1957); a ontologia e

3 Devido à multiplicidade de formas propostas - às vezes incompatíveis - para classificar os organismos, o problema da espécie surge, aponta Bartlett (2015). Até o momento, não existe uma forma única e adequadamente geral de definir o significado de "espécie"; a multiplicidade de formas distintas de classificar os organismos reflete uma multiplicidade correspondente de formas distintas 
metafísica da espécie (SLATER, 2013; STAMOS, 2003); a evolução das espécies; a conservação das espécies (CLARIDGE; DAWAH; WILSON, 1997); debate entre espécies e teorias sistemáticas (WHEELER; MEIER, 200o); entre outras questões apontadas em uma infinidade de artigos, livros e congressos.

Reconhecendo a nossa limitação diante do universo de conteúdos que se apresentam para debater o conceito de espécies, no presente texto buscamos refletir o conceito de espécie, com foco no estatuto ontológico do Conceito Biológico de Espécie e sua presença em livros didáticos do Ensino Médio. Discutimos também a maneira pelo qual é orientada a discussão do conceito de espécie nos documentos oficiais de Educação, buscando evidenciar a articulação do Conceito Biológico de Espécie aos aspectos de conservação e biodiversidade.

\section{A etimologia da palavra Espécie}

A palavra "espécie" é a uma forma latinizada da palavra grega "eidos", que foi apropriada por Platão, sendo que a palavra grega comum eidos significa "o aspecto de uma coisa" (STAMOS, 2003). Os termos traduzidos como "espécie" (eidos; grego:

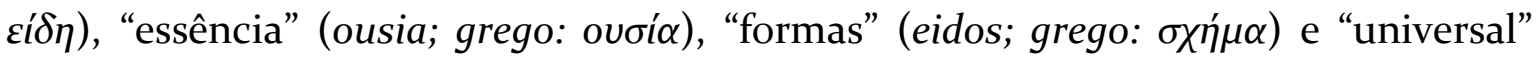

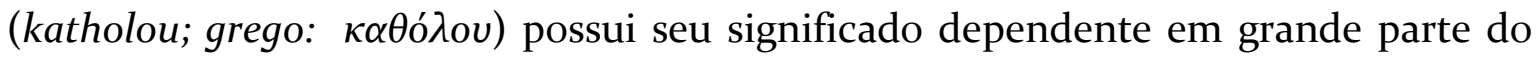
contexto que Aristóteles traz na discussão dos seus escritos, sendo "forma" sinônimo de "espécies" (STAMOS, 2003).

Como as palavras são derivadas etimologicamente de termos antigos ou traduzem palavras em outros idiomas, não ocorre imediatamente que sejam os mesmos termos com igual intenção ou extensão, como aponta Wilkins (2010). As palavras eidos e espécies são expressas por muitos autores, como: formas, tipos (types), tipos (kinds), espécies, espécies biológicas, classes, indivíduos, e coleções artificiais ou naturais (WILKINS, 2010) ${ }^{4}$. Atualmente o termo espécie é utilizado na biologia de diferentes formas e contextos, representando uma diversidade de conceitos (BARTLETT, 2015). Dependendo do conceito de espécie utilizado, é possível discutir aspectos específicos de determinadas áreas de pesquisas, do ensino ou ainda de compreensão prática dos fenômenos naturais.

Wilkins (2010), ao discutir a obra "On the Origin of Species" de Charles Darwin (1859), aponta que o livro "mudou a maneira de cada cientista olhar para as espécies" (WILKINS, 2010, p. 130, tradução nossa), justificando o ativo debate que acontece acerca desse conceito. Como afirma Neto (2011), com o Darwinismo, as espécies deixam de ser pensadas no sentido de correspondência a uma essência, no

de identificar e reconhecer propriedades que podem ser especificas, de modo a agrupar organismos em classes separáveis (BARTLETT, 2015).

4 Para uma leitura com maior profundidade do Conceito de Espécie, recomendamos conferir a obra "Species: a history of idea" elaborada por Wilkins (2010), onde faz apontamentos partindo da Era Clássica, desde Platão à Aristóteles, fazendo uma ponte na Era Medieval, chegando até o nascimento da Ciência Moderna. 
qual os organismos deveriam ter determinadas características (essência) para fazer parte de uma dada espécie. Para o autor, rompe-se com essa ideia para se pensar em termos de variabilidade e evolução.

Considerando a grande diversidade de entendimento do conceito de espécie, no próximo tópico, será explorada a definição de espécie como classe e como indivíduo, dois modos distintos de pensamento estabelecidos no debate metafísico da natureza da espécie. Em seguida, iremos apresentar um dos conceitos mais utilizados na Biologia, o Conceito Biológico de Espécie, discutindo se esse conceito se aproxima da compreensão ontológica de espécie como indivíduos. Depois, argumentamos como esse conceito é importante para compreender a diversidade biológica, ressaltando também suas limitações.

\section{Espécies como Classes ou como Indivíduos}

De acordo com Copi5 (1954) apud Stamos (2003), a ciência moderna procura conhecer as essências reais das coisas, e seus sucessos crescentes parecem se aproximar gradativamente dessa meta. Contudo, podemos discutir se existe a possibilidade de um conceito se aproximar gradativamente da essência ou da forma que o mundo de fato é, já que toda construção científica é permeada pela subjetividade e um aparato cognitivo que é próprio da espécie humana (GARDNER, 2003). Assim, entendemos que apesar de considerarmos uma definição de um fenômeno natural como coesa, sistematizada e útil para o desenvolvimento de ciência e tecnologia, a mesma será sempre uma verdade provisória, como é apontado em Popper ([1934] 1974). Isso não impede, todavia, que teorizemos acerca da existência de entidades reais na natureza, ou seja, que busquemos nos aproximar de sua ontologia (STAMOS, 2003). Nesse sentido, tentativas de determinar e delimitar espécies como entidades existentes na natureza, com origem evolutiva e por meio de um processo de diversificação das espécies, aproxima-se de uma construção ontológica do conceito de espécies (STAMOS, 2003, p. 4). O que se deseja, portanto, é um critério ou um conjunto de critérios pelos quais as coisas aparentemente reais possam ser julgadas (STAMOS, 2003, p. 10).

Dentro desse aspecto ontológico da Biologia, segundo Neto (2011), uma questão que surge é se as espécies constituem classes/tipos naturais ou indivíduos. Para o autor, no entendimento das espécies como classes ou tipos naturais, buscarse-ia:

[...] a existência de um conjunto de características qualitativas essenciais, presentes de modo necessário e suficiente em cada um dos seus membros. Essas características seriam causalmente

5 COPI, Irving M. Essence and Accident. The Journal of Philosophy, v. 51, p. 706-719. 1954. Reimpressão in: Stephen P. Schwartz (ed.). Naming. Necessity and Natural Kinds. Ithaca: Cornell University Press. 
responsáveis pela mencionada similaridade, o que explica o fato das propriedades de uma espécie poderem ser projetadas para cada um de seus organismos, ainda que não os identifiquemos. Notemos também que, se as espécies são definidas por características qualitativas fenéticas e/ou genéticas, é de se esperar que sua classificação seja baseada em tais características e não em relações genealógicas (NETO, 2011, p. 191).

O entendimento de espécies como classes, de acordo com Stamos (2003), obtém o seu maior apelo, devido ao fato de permitir a realidade de espécies assexuadas (uniparentais). As espécies assexuadas exibem um grau de coesão fenotípica, geralmente semelhante às espécies sexuais (biparentais) e são do ponto de vista taxonômico espécies genuínas. As espécies assexuadas também parecem igualmente habitar nichos, desempenhar papéis em cadeias alimentares, estarem sujeitas a controle biológico e evoluir (STAMOS, 2003). Incluir as espécies assexuadas entre espécies genuínas parece não apenas natural e legítimo, mas também proporciona direção e justificativa aparente para a visão de que espécies são classes de algum tipo, afirma Stamos (2003). Por outro lado, a visão que concebe espécies como indivíduos vai além do reconhecimento de similaridades entre grupos de organismos, entendendo as espécies como totalidades concretas e coesas, delimitadas no espaço e no tempo, ou seja, são espaço-temporalmente restritas, de modo que nomes de espécies são nomes próprios (GHISELIN, 1997). Este "é um afastamento radical e revolucionário da visão tradicional" (STAMOS, 2003, p. 181, tradução nossa) de espécies como classes, que por sua vez, são espaçotemporalmente irrestritas (GHISELIN, 1997). Como demonstra Neto (2011, p. 191), por meio de um olhar darwinista, as espécies passam a ser tratadas como entidades históricas que persistem no tempo mediante os processos de hereditariedade, propiciando a compreensão das "relações genealógicas entre organismos e populações de diferentes gerações". Nesse sentido, entender uma espécie como indivíduo é compreendê-la como uma entidade real que tem uma origem evolutiva, uma existência espaço-temporal (NETO, 2011) e que pode, por exemplo, ser extinta (GHISELIN, 1997).

Neto (2011) discutindo a ideia de espécie como indivíduos apresentada pelo filósofo David Hull (1976) destaca a diferença entre indivíduos e tipos naturais, já que indivíduos seriam compostos por partes e as classes/tipos por membros. As partes dos indivíduos não têm a necessidade de terem similaridades (ou seja, de uma essência que os tornaria membro de uma classe/tipo), mas estão unidos por meio de uma relação de coesão, tanto temporal como espacial (HULL, $1976^{6}$ apud NETO, 2011). Nesse contexto, na discussão da tese de individualidade, Ghiselin (1997) aponta que no sentido biológico usual, indivíduo é sinônimo de organismo, mas o termo ontológico é muito mais amplo. Embora, no sentido ontológico, todos

6 HULL, David. Are species really individuals? New Haven: Systematic Zoology, n. 25, p. 174-191, 1976. 
os organismos sejam indivíduos, nem todos os indivíduos no sentido ontológico serão indivíduos no sentido biológico usual (GHISELIN, 1997), pois seria possível na ontologia dar individualidade a partes e/ou membros de organismos e elementos inanimados, entretanto, para a individualidade ontológica no sentido biológico, apenas o organismo vivo e completo interessa.

Embora o pensamento não seja dominante tanto quanto a ideia de espécies como classes, a visão de espécies como indivíduos é influente em relação ao estatuto ontológico das espécies (STAMOS, 2003). Stamos (2003) aponta que a controvérsia entre a visão de espécies como classes e indivíduos iniciou com o trabalho de Michael Ghiselin em 1974, no qual alega que o seu posicionamento abordado na obra "Metaphysics and the Origin of Species" explicitou o que a maioria de naturalistas e biólogos manteve implícito nos dois últimos séculos.

O precursor mais claro da tese da individualidade moderna foi Julian Huxley (1887-1975), ao qual afirmou que: "se a evolução ocorreu" "as espécies não são mais constantes ou permanentes do que os indivíduos" (STAMOS, 2003, p. 186, tradução nossa). Ernst Mayr também foi um dos mais importantes precursores para a tese da individualidade, graças as suas contribuições ao Conceito Biológico de Espécie e a tese de individualidade que se desenvolveu a partir deste (STAMOS, 2003). Contudo, a visão de espécies como indivíduos, nega aos organismos assexuados qualquer status de espécie, pois afirma Ghiselin (1997) uma "espécie precisa ser uma população [...]”. Stamos (2003) aponta que nesse caso, a espécie como população se dá no sentido amplo de uma comunidade reprodutiva. Desta maneira, a ontologia de Ghiselin (1997) aponta a necessidade de dois tipos de indivíduos, um para espécies sexuais e outro para espécies assexuadas e táxons superiores. Na tese da individualidade, também se afirma que a extinção das espécies é necessariamente para sempre (STAMOS, 2003), assim, em um contexto biológico significa dizer que não é possível que uma espécie volte a existir depois que o último espécime morre (GHISELIN, 1997).

Na tese da espécie como individualidade, um indivíduo (espécies) ocupa uma posição definida no espaço e no tempo, portanto, possui começo e fim. E embora possa passar de um lugar para outro, é preciso haver uma continuidade no espaço e no tempo (GHISELIN, 1997). A ideia de espécie como individualidade está associada com a percepção de história evolutiva, genealógica e com o processo de especiação. Nesse sentido, o Conceito Biológico de Espécie está estreitamente vinculado a essa perspectiva.

Para Folgera; Marcos (2013), a tese de espécies como indivíduos não é isenta de problemas, por exemplo, as espécies em determinados aspectos parecem não se encaixar nas características do caso que é oferecido como paradigma, ou seja, da individualidade das espécies. Há problemas relacionados a espécies-irmãs e em casos de poliplóidia (quando há variação natural ou induzida no número de 
cromossomos, ou seja, existe a presença de pelo menos dois genomas no núcleo).

Espécies podem parecer discretas para o observador casual, sendo possível notar que alguns organismos são muito semelhantes, de outro modo, organismos membros da mesma espécie podem ser indistinguíveis para o olho humano não treinado (HOLTER, 2009). Por outro lado, na Biologia Evolutiva as espécies são tidas como unidade de evolução, ou seja, cadeias genealógicas ao longo das quais as mudanças evolutivas são acumuladas (NETO, 2013). "A tese individualista parte dessa atribuição e conclui que as espécies são indivíduos, e não tipos naturais" (NETO, 2013, p. 81).

Ainda que a tese de espécie como entidades individuais tenha limitações, compreendemos que ela busca descrever as relações genealógicas e evolutivas, apresentando uma ontologia histórica e que pode propiciar a compreensão de processos complexos como a diversificação e manutenção das espécies. Embora, não se tenha um conceito universal de espécie, entendemos que o Conceito Biológico de Espécie, amplamente utilizado e discutido, pode ser mais bem representado quando entendido, ainda que de maneira parcial, como espécie como entidades históricas, concretas e individualizadas. Nesse contexto, apresentamos as aproximações do Conceito Biológico de Espécie com a tese da individualidade a seguir.

\section{O Conceito Biológico de Espécie}

O Conceito Biológico de Espécie foi defendido amplamente por Ernst Mayr (1920-2004), ornitólogo alemão radicado em território estadunidense e definido como "populações geneticamente distintas e isoladas reprodutivamente de outros grupos" (MAYR, 1940, p. 256, tradução nossa). Os critérios básicos de definição de uma espécie nesse conceito, de acordo com Mayr (1940) são: 1) caracteres morfológicos; 2) distinção genética; 3) ausência de hibridação. Mayr (200oa) afirma que não interessa o grau de diferenciação morfológica, e sim as relações genéticas, portanto as espécies não podem ser definidas como classes, mas como entidades concretas de indivíduos que podem ser descritas e delimitadas. Logo, como afirma Mayr (200oa), uma espécie biológica é um agrupamento de genótipos - pool gênico - em harmonia e quando ocorre um intercruzamento o desequilíbrio acontece. "Os mecanismos de isolamento são propriedades biológicas de indivíduos que impedem o cruzamento de populações que são real ou potencialmente simpátricas" (MAYR, 1982, p. 274, tradução nossa). No entanto, o status de espécie é uma propriedade de populações e não de indivíduos (no sentido de seres vivos) e uma população não perde seu status de espécie quando um indivíduo hibridiza (MAYR, 200oa).

O Conceito Biológico de Espécie é amplamente aceito na Biologia Evolutiva, estabelecendo uma abordagem epistemológica específica em relação à teoria

7 As populações simpátricas são duas espécies ou populações que ocorrem em um mesmo local e que se tornam duas ou mais espécies distintas (RIDLEY, 2006). 
evolutiva (CRACRAFT, 1987). A abordagem epistemológica adotada pelos biólogos aponta que: "as espécies são unidades reprodutivamente dispersas ou isoladas; a especiação é a origem das unidades reprodutivamente isoladas; a genética é a alteração do genoma que provoca o isolamento reprodutivo; o isolamento reprodutivo é a principal consequência do processo evolutivo" (CRACRAFT, 1987, p. 338, tradução nossa). No entanto, Mishler; Theriot (2000) afirmam que o Conceito Biológico de Espécie carece de uma epistemologia coerente, uma vez que, a capacidade de cruzamento é o único critério epistemológico presente no conceito.

O Conceito Biológico de Espécie está focado claramente na ontologia das espécies, como afirma Mayr (200oa), de que os indivíduos são semelhantes porque pertencem a mesma espécie e não que os indivíduos pertencem a mesma espécie porque são semelhantes, ou seja, a semelhança emerge da história evolutiva do grupo, não sendo uma classificação abstrata e artificial. Entretanto, o reconhecimento de táxons de espécie é subjetivo e o uso da categoria subespécie pode indicar níveis de especiação incompleta ao se trabalhar com variação morfológica em populações alopátricas ${ }^{8}$ (KISCHLAT, 2005).

De acordo com Stamos (2003), Ernst Mayr aceitava parcialmente a tese da individualidade conforme foi proposta na obra "Metaphysics and the Origin of Species" de Michael Ghiselin (1997), pois seria bastante contraintuivo entender as espécies como indivisíveis assim como entendemos os seres vivos em geral. Entretanto, parece que a razão mais forte, segundo Stamos (2003, p. 195), é que "a natureza da organização (coesa) é muito diferente... pois a interação de partes de uma espécie é para a maioria de seus membros solta e indireta, consistindo apenas na propensão para a troca genética".

Independente do entendimento do Conceito Biológico de Espécie ser considerado como espécie-indivíduo, existem críticas realizadas para esse conceito. Dentre as críticas, Kischlat (2005, p. 16) compila seis importantes tópicos encontrados na obra Species concepts and phylogenetic theory - a debate de Wheeler; Meier (2000):

1) incompatibilidade com espécies assexuadas; 2) observação da presença ou ausência de intercruzamento pode até ser impossível de se realizar, não sendo possível aplicar a formas extintas; 3) delimitação de espécie reflexo de uma situação não-dimensional ${ }^{9}$; 4) intercruzamento e coesão de uma população representam uma característica herdada, logo não representa critérios de distinção,

8 Populações alopátricas é a definição dada para populações biológicas que ficam isoladas por barreiras geográficas ou alocrônicas (espécies de sucessão) (RIDLEY, 2006).

9 A situação não-dimensional do Conceito Biológico de Espécie é representado quando se enfatiza a proteção de um pool gênico harmonioso. A proteção de um pool gênico é estritamente biológico e possui significado apenas onde o pool gênico de uma espécie entra em contato com o pool gênico de outra espécie, em uma determinada localidade e em um determinado tempo (o que representa uma situação não-dimensional) (KISCHLAT, 2005, p.8). 
seja de espécies ou populações; 5) o reconhecimento de espécie como um mecanismo de proteção do equilíbrio e harmonia do genótipo é determinístico e impõe que se assuma espécie como um estágio final de preservação destes genótipos quanto à misturas posteriores; 6) o conceito de espécie deve permitir a avaliação da biodiversidade e a utilização da categoria subespécie acaba por reduzir o número de unidades da biodiversidade (KISCHLAT, 2005, p.16).

Tais críticas apontadas acima estão presentes na obra Species concepts and phylogenetic theory - a debate, e são apresentadas por diversos autores, proponentes de conceitos de espécies, tais como: o Conceito Evolutivo; o Conceito Filogenético versão Diagnóstico (versão de WHEELER; PLATNICK, 200o); o Conceito Filogenético versão Monofilia (versão de MISHLER; THERIOT, 200o). Essas críticas são refutadas por Ernst Mayr no capítulo 12 da mesma obra (WHEELLER; MEIER, 200o). Mayr (200ob) aponta ainda, que um conceito de espécie deve ser baseado na descrição do papel que as espécies desempenham no mundo vivo, fato que nem as definições dos conceitos evolutivas e filogenéticos conseguem explicar (MAYR, 200ob).

As respostas apresentadas por Mayr (200ob) para rebater as críticas das fragilidades destacadas acima ao Conceito Biológico de Espécie são brevemente aqui expostas: 1) o Conceito Biológico de Espécie simplesmente não se encaixa em organismos de reprodução assexuada, pois as agamoespécies não formam populações como definido pelos biólogos; não estão sujeitas a recombinação genética, logo os pools gênicos desses clones não necessitam de proteção por mecanismos de isolamento; 2 e 3) é necessário um critério para demarcação dos táxons de espécies, principalmente para populações isoladas, sendo necessário tanto para a dimensão temporal quanto para a geográfica. É explicado também, que a restrição geográfica habitual do Conceito Biológico de Espécie facilita a aplicação do conceito aos fósseis. O Conceito Biológico de Espécie, explica Mayr (200ob), é válido apenas na situação não-dimensional, pois o corte de uma linhagem filética contínua em espécies, deve ser feito por inferência, ou seja, a partir da subjetividade do biólogo taxonomista; 4) Mayr (20oob) afirma que não utiliza o conceito de coesão reprodutiva, mas afirma, que todas as populações de uma espécies são capazes de cruzar. Aponta que o status de espécie de uma determinada população com relação a uma população diferente pode ser testado, somente onde elas coexistem no espaço e no tempo; 5) da crítica de espécie como um dispositivo para a proteção de genótipos harmoniosos, Mayr aponta que as espécies são o produto da história evolutiva, sendo o Conceito Biológico de Espécie baseado em um cenário explicativo. É apontado ainda, que as diferenças morfológicas não são critérios de espécie, portanto, as diferenças na variabilidade são irrelevantes para o conceito de espécie; 6) chamar subespécies bem definidas como espécies, de acordo com Mayr, é voltar a um conceito de espécies estritamente tipológico. Tratar espécies 
incipientes e raças geográficas da mesma maneira que espécies genuínas duplicaria o número de espécies, com base em critérios que não possuem nada em comum com o significado biológico das espécies.

Apesar das limitações que o Conceito Biológico de Espécie encontra, o mesmo é bastante utilizado e importante para organismos com reprodução sexuada, uma vez que, o critério reprodutivo é uma das formas de identificação das espécies por meio desse conceito. Como o Conceito Biológico de Espécie está estreitamente relacionado às explicações dos mecanismos de especiação e da descrição da história evolutiva dos seres vivos, entendemos que ele se aproxima de uma descrição ontológica que busca explicar a origem e diversificação dos seres vivos a partir do isolamento reprodutivo. Além disso, esse conceito pode ser considerado (ainda que com ressalvas) próximo à ideia de espécie-indivíduo, já que as espécies possuiriam certa coesão no tempo e no espaço, embora a sua descrição se dê em vias de não restrição de tempo e espaço. Esse conceito é, portanto, importante para superar a ideia de espécie como classes e pensar na biodiversidade em termos evolutivos.

No tópico seguinte apresentamos como os documentos oficiais de Educação e os Livros Didáticos tratam a abordagem do conceito de espécie e da diversidade biológica, fazendo articulação com o debate travado até esse momento e com o Ensino de Biologia.

\section{Os Documentos de Educação, os Livros Didáticos e o Conceito de Espécie no Ensino de Biologia}

De acordo com as Orientações Curriculares para o Ensino Médio (BRASIL, 2006a), o Ensino de Biologia deve proporcionar ao estudante a possibilidade de participar dos debates atuais que necessitam de conhecimentos biológicos bem como reconhecer a importância da biodiversidade e a influência que esta gera na qualidade de vida humana. O Ensino de Biologia precisa aceitar o desafio de formar o "[...] indivíduo com um sólido conhecimento biológico e com raciocínio crítico" (BRASIL, 2006a, p. 17).

Dentre os seis temas estruturantes do currículo de Biologia apresentados pelos Parâmetros Curriculares Nacionais + $(\mathrm{PCN}+)$ (BRASIL, 2002), a temática Origem e Evolução da vida é um tema central no Ensino de Biologia, devendo constituir base para a discussão das outras cinco temáticas (BRASIL, 2006b) presentes no documento. A diversidade biológica, segundo o documento, deve ser tratada em todos os níveis, discutindo-se a "[...] diversidade dos ecossistemas, das populações, das espécies e dos genes" (BRASIL, 2006b, p.23). O estudante deve ter que compreender que as mutações são a fonte primária da variabilidade, necessitando ser estimulado a discutir as questões envolvidas sob a ótica da evolução por meio da intervenção humana, como, a seleção artificial e o surgimento e perda de espécies (BRASIL, 2006b). 
Em relação aos temas estruturadores do Ensino de Biologia, os $\mathrm{PCN}+\mathrm{EM}$ (BRASIL, 2002) apresenta como um dos temas "Diversidade da vida", no qual os conteúdos de zoologia, botânica e ciências ambientais são entendidos como fundamentais para a compreensão do processo de diversificação da vida a partir de uma origem comum e para apresentar dimensionamentos aos problemas voltados a biodiversidade (BRASIL, 2002).

O entendimento da biodiversidade proporciona meios para avaliar a riqueza de determinada área e pode ser utilizada de base para propostas conservacionistas (KISCHLAT, 2005), que culminam na elaboração de políticas públicas, podendo levar, por exemplo, a criação de parques e unidades de conservação. Esta biodiversidade em questão é consequência direta da aplicação de um conceito de espécie (KISCHLAT, 2005). Nesse sentido, os hotspots ${ }^{10}$ de biodiversidade são uma das abordagens mais influentes para definir áreas de prioridade de conservação, correspondendo a uma área terrestre de difícil substituição e importante vulnerabilidade (ZACHOS, 2016). Nesse cenário, a biodiversidade é frequentemente quantificada em estudos evolutivos, tendo como unidade básica, a espécie (ZACHOS, 2016).

No Ensino de Biologia, previsto no PCN + EM, propõe-se que o estudante seja conduzido a: conhecer e utilizar os critérios de classificação, as regras de nomenclatura e as categorias taxonômicas atuais; reconhecer as características dos cinco reinos, atualmente organizado em domínios e identificar especificidades relacionadas com as condições ambientais; construir árvores filogenéticas para representar relações de parentesco entre os seres vivos (BRASIL, 2002). Desse modo, explora-se a evolução das espécies, considerando seus mecanismos evolutivos, como a mutação, recombinação gênica e a seleção natural, possibilitando ao estudante o contato com conhecimentos de evolução biológica.

A Base Nacional Comum Curricular (BNCC) para o Ensino Médio (BRASIL, 2018), aprovada pelo Conselho Nacional de Educação em 4 de dezembro de 2018 e homologado pelo Ministério da Educação em 19 de dezembro de 2018, não indica as disciplinas a serem abordadas, mas quatro áreas do conhecimento. Uma das áreas do conhecimento é Ciências da Natureza e suas Tecnologias. Nesse documento, cada área do conhecimento apresenta competências específicas, sendo que para cada competência é identificado um conjunto de habilidades (BRASIL, 2018). Para o Ensino Médio é visualizada a unificação das temáticas "Vida e Evolução" e "Terra e Universo", promovendo a compreensão ampla dos processos abordados nas duas unidades temáticas, por parte dos estudantes. Dentre as competências específicas, por exemplo, a competência número Dois, diz que o estudante, deve analisar e

$10 \mathrm{O}$ conceito de hotspots de biodiversidade é caracterizado por áreas em que são encontradas grandes quantidades de espécies endêmicas, ainda associado a altas taxas de destruição dos habitats (MYERS et al., 2000). 
utilizar interpretações sobre a dinâmica da Vida, Terra e Cosmos para elaborar argumentos, realizar previsões a respeito do funcionamento e a evolução dos seres vivos (BRASIL, 2018). Ainda na competência número Dois, os estudantes podem mobilizar conhecimentos relacionados com a origem da vida, evolução biológica, biodiversidade, origem e extinção das espécies e outros. Para a habilidade Dois, os estudantes necessitam interpretar as formas de manifestações da vida e considerar os seus diferentes níveis de organização. Na habilidade Seis, é apontada a importância de o aluno justificar e compreender a importância da preservação e conservação da biodiversidade, avaliando os efeitos da ação humana e das políticas ambientais, como meio de assegurar a sustentabilidade do planeta (BRASIL, 2018).

A articulação do conceito de espécie com conteúdos do eixo ecológico e evolutivo se faz bem presente nos documentos de Educação, assim, consideramos a importância de se apresentar o Conceito Biológico de Espécie aos estudantes, associando-o ao processo de diversificação das espécies, à teoria evolutiva e à compreensão da biodiversidade. Fazendo-se necessário que o Conceito Biológico de Espécie esteja articulado à compreensão histórica dos seres vivos, aos mecanismos de especiação e da compreensão da coesão espaço-temporal das espécies. Nesse sentido, é importante identificar as espécies como entidades históricas (o que se aproxima da compreensão de espécies como indivíduos), concretas e que podem ser extintas. Essa visão ontológica das espécies pode propiciar a reflexão a respeito da manutenção das espécies, o cuidado com as intensas modificações ambientais que podem levar a essas extinções, entre outros aspectos. Ressaltamos também que é importante ao trabalhar o Conceito Biológico de Espécie mostrar os limites desse conceito, por exemplo, na utilização do mesmo para espécies assexuadas, e indicar a existência de uma diversidade de conceitos de espécies que são utilizados de acordo com as áreas de pesquisas.

Compreendido a maneira como é indicado nos documentos oficiais de Educação o ensino de temáticas que discutem as questões de espécies, a biodiversidade, as relações da vida e a sua evolução, buscamos conhecer como acontece à apresentação destes conteúdos nos livros didáticos de Biologia.

Por meio da conferência do sumário dos livros didáticos das dez coleções de Biologia indicados pelo Programa Nacional do Livro Didático (PNLD/2018), foi possível identificar que a temática da "diversidade biológica" geralmente é tratada na primeira unidade do Segundo volume das coleções. Diversos títulos recebem o capítulo que trata da temática, comumente abordando a classificação dos seres vivos, a sistemática e a biodiversidade de organismos presente no planeta. Dentre as temáticas trazidas no capítulo de biodiversidade, sistemática e classificação, o conceito de espécie é um item “obrigatório" a ser abordado, seja de maneira genérica e resumida, ao ser apresentada uma ideia geral do significado do que se trata o conceito de espécie, ou de modo amplo, adotando a explicação de 
determinado conceito de espécie.

Conforme apontado por Silva (2018), o Conceito Biológico de Espécie é o conceito mais abordado nos livros didáticos de Biologia, o que pode reforçar nos estudantes o entendimento de um conceito de espécie único, muito embora, seja apresentado nos livros didáticos que existem diversas alternativas de conceitos de espécies na literatura acadêmica (SILVA, 2018).

As espécies são abordadas, por exemplo, nos conteúdos de botânica e zoologia, nos conteúdos de especiação, e em outros capítulos, nos livros didáticos destinados aos alunos e professores (MENDONÇA, 2016). Porém, geralmente são apresentados exemplos com características universais, conforme apontado por Fonseca (2007), indicando espécies de ambientes diferentes daqueles da comunidade escolar. Esses podem ser exemplos consagrados pela literatura e que possivelmente ilustram adequadamente o conteúdo a ser ensinado, entretanto, é recomendado trazer exemplos da fauna e flora local para a sala de aula, para a valorização dos recursos biológicos regionais (FONSECA, 2007). Deste modo, o professor estará indicando aos alunos que os fenômenos apresentados nos livros didáticos não acontecem apenas a centenas de quilômetros de sua casa, mas também está ocorrendo na região em que vivem. Por meio da contextualização do conteúdo a ser ensinado, o professor tem a oportunidade de aproximar os alunos dos fenômenos naturais, ao situar e relacionar os conteúdos a diferentes contextos de sua produção, apropriação e utilização (KATO; KAWASAKI, 2011).

O Conceito Biológico de Espécie é o conceito mais abordado nos livros didáticos de Biologia, como já apontado em Silva (2018), portanto, é um conceito que estará presente no cotidiano escolar, desta maneira, é importante o planejamento adequado das aulas para que satisfaça as necessidades de seus alunos (ROJO, 2001) e conduza os estudantes ao debate e a reflexão a respeito das espécies e sua importância na natureza.

Em relação a ontologia do conceito de espécie, perguntas como "o que é uma espécie?” ou que “que tipo de entidade é uma espécie?” pode guiar a discussão no Ensino de Biologia. Entre as diversas possibilidades de responder a estes questionamentos, o professor pode se apropriar do Conceito Biológico de Espécie, que goza de ampla aceitação na Biologia (CRACRAFT, 1987). Embora haja críticas pertinentes a sua estrutura teórica e aplicação, pode ser o início da compreensão do complexo mundo da sistemática e da taxonomia. O Conceito Biológico de Espécie é naturalmente ontológico (KISCHLAT, 2005) e se aproxima do entendimento de que as espécies são indivíduos, aceitando em parte a premissa de individualidade do paradigma atual do estatuto ontológico das espécies. Contudo, em alguns momentos outros conceitos de espécies podem ser necessários para estudar os processos biológicos. 
Ao abordar a descrição e a preservação da diversidade de seres vivos no planeta, pode-se evidenciar os limites e críticas ao Conceito Biológico de Espécie elaboradas, em geral, por proponentes de outros conceitos (WHEELER; MEIER, 200o). É importante também apresentar as refutações apresentadas pelo próprio Mayr (20oob) em relação às críticas recebidas. Compreender que os conceitos da ciência estão em debate, permite ao aluno construir uma ideia de que a ciência apresenta ideias divergentes e que nem sempre é possível estabelecer um conceito que seja suficiente para representar uma diversidade de situações, processos e fenômenos naturais (MELLO ARRUDA et al., 2005).

O Conceito Biológico de Espécie pode apresentar uma resposta no Ensino de Biologia ao problema da descrição da diversidade e para a preservação da biodiversidade, sem provocar, como já apontou Mayr (2000a), um aumento inflacionário no número de espécies, como aconteceria com alguns conceitos filogenéticos. Contudo, o Conceito Biológico de Espécie não pode ser utilizado para organismos assexuados (WHEELER; MEIER, 200o), dessa forma, poderia ser apresentado aos alunos outro conceito de espécie, por exemplo, o Conceito de Espécie Agamoespecífico, que reconhece linhagens e pode descrever a diversidade de organismos assexuados (TURESSON, 1929).

No caso de organismos híbridos, como é o caso de certos mamíferos, pode-se ressaltar que são organismos resultantes, muitas vezes, de cruzamentos artificiais, produzidos pela interferência humana, que busca selecionar características que the seja útil (CATANI, 2016). No entanto, essa é apenas uma pequena parcela de um todo, pois, a maioria dos organismos híbridos na natureza ocorre sem ação humana. Esse é um exemplo que desafia o Conceito Biológico de Espécie, uma vez que afirma que quando numa espécie, um organismo hibridiza, a espécie da qual originou o organismo híbrido não perde seu status de espécie (MAYR, 200oa), no entanto, o organismo híbrido permanece em uma forma de limbo, sem ser caracterizado como uma nova espécie.

Muitas plantas se reproduzem sexuada e assexuadamente, de modo que alguns indivíduos são resultados de recombinação gênica, enquanto outros são resultantes de divisões mitóticas, ou seja, são geneticamente idênticos à planta-mãe (RAVEN; EICHHORN; EVERT, 1996). Grupos de plantas com sobrevida longa podem formar híbridos férteis, por sua vez, os híbridos entre plantas herbáceas geralmente são estéreis ou ocorrem apenas com dificuldade. Portanto, como afirma Raven; Eichhorn; Evert (1996), é preferível evitar o uso exclusivo do critério de isolamento reprodutivo para definir espécies de plantas, ou seja, esse é um fator limitador para a utilização do Conceito Biológico de Espécie.

Outro caso em que o Conceito Biológico de Espécie não é adequado é para a identificação de espécies fósseis (WHEELER; MEIER, 200o). Reconhece-se a importância dos fósseis, pois consistem de registros em rochas da existência de vida 
no passado do planeta (RIDLEY, 2006), sendo de grande importância para a discussão de temas paleontológicos inseridos em tópicos mais abrangentes, como por exemplo, a evolução orgânica, a sistemática biológica e a origem da vida (ARAÚJO JÚNIOR; OLIVEIRA PORPINO, 2010). Neste caso, pode-se recorrer a formas morfológicas, principalmente no registro paleontológico, adotando critérios de definição de paleoespécies, presentes no Conceito de Espécies Sucessórias" (ZACHOS, 2016).

Quando uma espécie está se dividindo para formar duas espécies, ao longo do tempo ocorre o processo de divergência, e em determinados momentos, as espécies filhas adquirem propriedades (entendidos como critérios de espécie) que as tornam distinguíveis da espécie-mãe (DE QUEIROZ, 2007). As propriedades podem ser: potencial cruzamento; reconhecimento de parceiro específico; mesmo nicho ecológico; monofilia ${ }^{12}$; formação de aglomerado (cluster) fenético ${ }^{13}$; e cluster genotípico, possibilitando que algum conjunto de propriedades se encaixe em alternativos conceitos de espécies (DE QUEIROZ, 2005b; DE QUEIROZ, 2007). Logo, uma espécie que se encontra em estágio intermediário de especiação não será reconhecida como uma espécie no Conceito Biológico de Espécie, sendo possivelmente reconhecida como uma subespécie, da espécie-mãe.

Uma das dificuldades da utilização de diferentes conceitos de espécies está no reconhecimento da própria biodiversidade de uma área, pois a contagem do número de espécies pode ser diferente, de acordo com o conceito de espécie utilizado (ZACHOS, 2016). Além disso, Zachos (2016) aponta que diferentes grupos de sistematas utilizarão diferentes conceitos de espécies, e, portanto, as entidades reunidas sob o nome de "espécie" poderão ser entidades muito diferentes, obscurecendo a quantificação da diversidade biológica. Essas explicações devem ser colocadas de forma acessível aos estudantes do Ensino Médio, de modo que compreendam a ciência como um processo em construção e que, muitas vezes, os conceitos de espécies são utilizados de formas diferentes dependendo da área do conhecimento biológico abordada.

\section{Considerações finais}

11 O Conceito de Espécies Sucessórias tem como referência George Gaylord Simpson (1961). A definição de espécies para este conceito se dá por "estágios anagenéticos (mudanças evolutivas dentro de uma única linha de descendentes através do tempo, ou seja, sem divisão de linhagem) arbitrários em formas morfológicas, principalmente no registro paleontológico (WILKINS, 2009, p. 198 apud ZACHOS, 2016).

12 A monofilia é definida como um táxon cujos membros possuem todos um ancestral comum e que compreende este ancestral e todos seus descendentes. Uma hipótese de monofilia é fundamentada na existência de traços derivados compartilhados (apomorfias) (ZACHOS, 2016).

$13 \mathrm{O}$ aglomerado fenético (cluster) se constitui de um conjunto de organismos semelhantes entre si e separados de outros organismos, baseados na similaridade geral, sendo a base do Conceito Fenético de Espécies (ZACHOS, 2016). 
No Ensino Médio, um dos conceitos de espécie que está mais presente é o do Conceito Biológico de Espécie. Apesar das limitações desse conceito e da necessidade do professor mostrar que existe uma diversidade de conceitos de espécies e que nenhum deles dará conta da totalidade dos processos biológicos, é importante que o professor faça reflexões a respeito do Conceito Biológico de Espécie de modo que leve aos alunos a compreender o processo de especiação e evolução dos seres vivos.

A abordagem do conceito de ontologia, embora pareça complexa, certamente se trazida para a sala de aula em um trabalho interdisciplinar, pode permitir aos alunos a compreensão da importância de se investigar e questionar a natureza do ser e a existência das espécies. A apresentação da concepção de espécies como indivíduos possibilita o entendimento da necessidade do debate evolucionista, sendo o professor responsável pela catalisação do debate entre seus alunos, conduzindo-os para a percepção de como pode ser influenciada a nossa visão da constituição dos conjuntos de seres vivos no planeta, as ações que delas são geradas e os impactos, sobretudo para a biodiversidade.

O Conceito Biológico de Espécie, em especial para os animais, é um importante conceito para pensar na biodiversidade e sua preservação, uma vez que, ajuda a entender o processo de diversificação dos seres vivos por meio da especiação e isolamento reprodutivo. Compreender que o fenômeno de especiação e a constituição de novas espécies são fenômenos únicos que acontecem ao logo da história do planeta, possibilita pensar na importância da manutenção das espécies e que as espécies extintas não podem ser recuperadas.

Compreende-se a importância da pluralidade de reflexões acerca dos conceitos de espécies e que sua discussão no Ensino de Biologia pode proporcionar aos estudantes o contato com discussões atuais. Desta forma, estaria se cumprindo com a orientação de proporcionar a participação nos debates atuais no qual os conhecimentos biológicos com ênfase evolucionista são requisitos básicos.

\section{Referências}

ARAÚJO JÚNIOR, Hermínio Ismael; OLIVEIRA PORPINO, Kleberson. Análise da abordagem do tema paleontologia nos livros didáticos de biologia. Rio de Janeiro: Anuário do Instituto de Geociências, v. 33, n. 1, p. 63-72, 2010.

BARTLETT, Steven James. The species problem and its logic: Inescapable ambiguity and framework-relativity. Ithaca: Cornell University Library. arXiv preprint arXiv:1510.01589, 2015. Disponível em: <https://arxiv.org/ftp/arxiv/papers/1510/1510.01589.pdf> Acesso em: 18 dez 2018.

BRASIL. Secretária de Educação Básica. Base Nacional Comum Curricular: Educação é a base. Ensino Médio. Brasília: Ministério da Educação. ed. 2017. 2018.

. Secretaria de Educação Básica. Orientações curriculares para o ensino médio Ciências da natureza, matemática e suas tecnologias. Brasília: Ministério da Educação, 
Secretaria de Educação Básica, (volume 2), 2006a. 135 p.

. Secretaria de Educação Básica. PCN + Ciências da Natureza, Matemática e suas Tecnologias. Orientações Educacionais Complementares aos Parâmetros Curriculares Nacionais. Brasília: Ministério da Educação, Secretaria de Educação Básica. 2006b.

Secretaria de Educação Básica. PCN + Ciências da Natureza, Matemática e suas Tecnologias. Orientações Educacionais Complementares aos Parâmetros Curriculares Nacionais. Brasília: Ministério da Educação, Secretaria de Educação Básica. 2002.

CATANI, André. Ser protagonista: biologia. 2o ano: ensino médio. Catani, André ...[et al.]; organizadora Edições SM; obra coletiva, concebida, desenvolvida por Edições SM; Bezera, Lia Monguilhott (ed. resp.). 3. ed. São Paulo: Edições SM, 2016.

CHEVALLARD, Ives. Pourquoi la transposition didactique? Communication au Séminaire de didactique et de pédagogie des matthématiques de I'IMAGE, Université Scientifique et Médicale de Grenoble. Paru dans les Actes de I’année 1981-1982, p. 167-194. 1982. Disponível em: <

http://yves.chevallard.free.fr/spip/spip/IMG/pdf/Pourquoi_la_transposition_didactique.pdf $>$ Acesso em: 07 nov. 2018.

CLARIDGE, Michael F.; DAWAH, Hassan A.; WILSON, Michael R. Pratical approaches to species concepts for living organisms. In: Species: The units of biodiversity. Claridge, M. F.; Dawah, H. A.; Wilson, M. R. (eds.). The Systematics Association Special Volume Series 54. London: Chapman e Hall. 1997. 1-13 p.

. Species: The units of biodiversity. Claridge, M. F.; Dawah, H. A.; Wilson, M. R. (eds.). The Systematics Association Special Volume Series 54. London: Chapman e Hall. 1997.

CRACRAFT, Joel. Species Concepts and the Ontology of Evolution. Philadelphia: Biology and Philosophy. n. 2, p. 329-346, 1987.

DE QUEIROZ, Kevin. A unified concept of species and its consequences for the future of taxonomy. San Francisco: Proceedings of the California Academy of Sciences. v. 56, n. 18, p. 196-215, 2005 a.

. Ernst Mayr and the modern concept of species. Whashington, D.C. Proceedings of the National Academy of Sciences, v. 102, n. suppl 1, p. 660o-6607, $2005 \mathrm{~b}$.

. Species concepts and species delimitation. New Haven: Systematic biology, v. 56, n. 6, p. 879-886, 2007.

FOLGUERA, Guillermo; MARCOS, Alfredo. El concepto de especie y los cambios teóricos en Biología. Ciudad de Mexico: Ludus Vitalis, vol. XXI, num. 39, p. 1-25, 2013.

FONSECA, Maria de Jesus da Conceição Ferreira. A biodiversidade e o desenvolvimento sustentável nas escolas do ensino médio de Belém (PA), Brasil. São Paulo: Educação e Pesquisa, v.33, n.1, p. 63-79, 2007.

GARDNER, Howard. A Nova Ciência da Mente: Uma História da Revolução Cognitiva. Howard Gardner; tradução de Claúdia Malbergier Caon; prefácio de Marcos Barbosa de Oliveira. São Paulo: Editora da Universidade de São Paulo. 3 ed. 2003.

GHISELIN, Michael T. Metaphysics and the origin of species. SUNY series in philosophy and biology. New York: State University of New York Press. 1997.

. "A radical solution to the species problem". New Haven: Systematic Zoology. n. 23, p. 536-544, 1974.

HOLTER, Brandon David. The ontology of species: a radically pluralistic perspective. Thesis 
Master of Arts in Philosophy. Department of Philosophy. Washington State University. August 2009. 78p.

HULL, David Lee. “Are species really individuals?”. New Haven: Systematic Zoology. n. 25, p. 174-191, 1976.

KATO, Danilo Seithi; KAWASAKI, Clarice Sumi. As concepções de contextualização do ensino em documentos curriculares oficiais e de professores de ciências. Bauru: Ciência $\mathcal{E}$ Educação, v. 17, n. 1, p. 35-50, 2011.

KISCHLAT, Edio-Ernst. Os conceitos de espécie: uma abordagem prática. Canoas: Caderno La Salle XI. v.2, no 1, 11-35, 2005.

MAYR, Ernst. A Defense of the Biological Species Concept. In: WHEELER, Q.D; MEIER, R. (Ed.). Species concepts and phylogenetic theory: a debate. New York: Columbia University Press. 20oob. p. 161- 166.

Populações, espécies e evolução. Tradução de Hans Reichardt. São Paulo: Ed.

Nacional, Ed. da Universidade de São Paulo. 1977.

. The Biological Species Concept. In: WHEELER, Q.D; MEIER, R. (Ed.). Species concepts and phylogenetic theory: a debate. New York: Columbia University Press. 200oa. p. 17-29.

The Growth of Biological Thought: Diversity, Evolution, and Inheritance.

Cambridge: Harvard University Press. 1982.

. The ontological status of species: scientific progress and philophical terminology.

Berlim: Biology \& Philosophy, v.2. p. 145-166, 1987.

. The Species Problem. Edited by Ernst Mayr. Publication n. 50. Whashington D.C.: Americam Association for the Advancement of Science. 1957.

. The speciation phenomena in birds. Chigaco: The American Naturalist. n. 74, p. 249-278. 1940.

MELLO ARRUDA, Sergio; UENO, Michele Hidemi; GUIZELLINI, Alessandra; PASSOS, Marinez Meneghello; MARTINS, João Batista. O pensamento convergente, o pensamento divergente e a formação de professores de ciências e matemática. Florianopólis: Caderno Brasileiro de Ensino de Física, v. 22, n. 2, p. 220-239, 2005.

MENDONÇA, Vivian Laurence. Biologia: o ser humano, genética, evolução. Volume 3: ensino médio. 3. ed. São Paulo: Editora AJS, 2016.

MISHLER, Brent D.; THERIOT, Edward C. The Phylogenetic Species Concept (sensu Mishler and Theriot): Monophyly, Apomorphy, and Phylogenetic Species Concepts. In: WHEELER, Q.D; MEIER, R. (Ed.). Species concepts and phylogenetic theory: a debate. New York: Columbia University Press. 2000. p. 44 - 54.

MYERS, Norman; MITTERMEIER, Russell A.; MITTERMEIER, Cristina G.; FONSECA, Gustavo A. B.; KENT, Jennifer. Biodiversity hotspots for conservation priorities. Londres: Nature, v. 403, p. 853-858, 2000.

NETO, Celso Antônio Alves. Considerações sobre o conceito de coesão na tese "espéciescomo-indivíduos” de David Hull. São Paulo: Filosofia e História da Biologia, v. 6, n. 2, p. 189209, 2011.

O estatuto ontológico das espécies biológicas na sistemática filogenética. Dissertação Mestrado. Departamento de Filosofia da Faculdade de Filosofia e Ciências Humanas. Belo Horizonte: Universidade Federal de Minas Gerais. 2013. 151 p. 
POPPER, Karl Raimund. A lógica da pesquisa científica. Tradução de Leonidas Hegemberg e Octanny Silveira da Mota. São Paulo: Cultrix, 1974.

RAVEN, Peter H.; EVERT, Ray F.; EICHHORN, Susan E. Biologia Vegetal. J. E. Kraus (Coord. trad.). Rio de Janeiro: Editora Guanabara Koogan, 5 a . ed., 1996.

RICHARDS, Richard A. The Specie Problem: A Philosophical Analysis. Cambridge:

Cambridge University Press. 2010.

RIDLEY, Mark. Evolução. Henrique Ferreira, Luciane Passaglia, Rivo Fisher (trad.). Porto Alegre: Artmed. 2006. 752 p.

ROJO, Roxane Helena Rodrigues. Modelização didática e planejamento: duas práticas esquecidas do professor. In: A formação do professor: perspectivas da lingüística aplicada. Campinas: Mercado de Letras, p. 313-335, 2001.

SILVA, Luciano Neves da. Os conceitos de espécies abordados em livros didáticos de Biologia. In: Anais...VI Simpósio Nacional de Educação. XXVII Semana Acadêmica de Pedagogia e I Mostra de Pós-graduação. Universidade do Oeste do Paraná. 2018. Disponível em: <https://midas.unioeste.br/sgev/eventos/visimposionacionaldeeducacao/anais $>$ Acesso em: 18 de set 2018 .

SLATER, Matthew H. Ares Specie Real? An essay on the metaphysics of species. Series New Directions of the Philosophy of Science. New York: Palgrave MacMillan. 2013.

STAMOS, David. The species problem: biological species, ontology, and the metaphysics of biology. Lanham: Lexington Books. 2003. 390 p.

TURESSON, Göte. Zur Natur und Begrenzung der Arteinheiten. (in German with an English summary). Londres: Hereditas, v. 12, p. 323-334 1929

WHEELER, Quentim Duane; MEIER, Rudolf (eds). Species concepts and phylogenetic theory: a debate. New York: Columbia University Press. 2000.

WHEELER, Quentim Duane; PLATNICK, Norman I. The Phylogenetic Species Concept (sensu Wheeler and Platnick). In: WHEELER, Q.D; MEIER, R. (Ed.). Species concepts and phylogenetic theory: a debate. New York: Columbia University Press. 2000. p. 55-69.

WILKINS, John S. Species: A History of the Idea. Species and systematics, v.1. Los Angeles: University of California Press. 2010.

ZACHOS, Frank E. Species concepts in biology: historical development, theoretical foundations and practical relevance. Cham: Springer, 2016.

Submissão: 10.10.2018 / Aceite: 20.12.2019 